\title{
Common Fixed Point Theorems for Compatible and Weakly Compatible Maps in G-Metic Spaces
}

\author{
Asha Rani ${ }^{*}$, Sanjay Kumar ${ }^{2}$, Naresh Kumar ${ }^{2}$, S. K. Garg ${ }^{2}$ \\ ${ }^{1}$ Department of Applied Sciences, B. M. Institute of Engineering and Technology, \\ Raipur, India \\ ${ }^{2}$ Departement of Mathematics, Deenbandhu Chhotu Ram University of Science and Technology, \\ Murthal, India \\ Email: asha.dahiya27@gmail.com,sanjaymudgal2004@yahoo.com,nks280@gmail.com,skg1958@gmail.com
}

Received February 20, 2012; revised August 27, 2012; accepted September 3, 2012

\begin{abstract}
In this paper first we prove common fixed point theorems for compatible and weakly compatible maps. Secondly, we prove common fixed point theorems for weakly compatible maps along with property (E.A.) and (CLRg) property respectively.
\end{abstract}

Keywords: G-Metric Spaces; Compatible Maps; Weakly Compatible Maps; Property (E.A.); (CLRg) Property

\section{Introduction}

In 1922, Banach proved a common fixed-point theorem which ensures under appropriate conditions, the existence and uniqueness of a fixed-point. This theorem has many applications, but suffers from one drawback - the definition requires that $T$ be continuous throughout $X$. There then follows a flood of papers involving contractive definition that do not require the continuity of $T$. This result was further generalized and extended in various ways by many authors. This theorem provides a technique for solving a variety of applied problems in mathematical sciences and engineering.

In 1963, Gahler [1] introduced the concept of 2-metric spaces and claimed that a 2-metric is a generalization of the usual notion of a metric, but some authors proved that there is no relation between these two functions. Geometrically, a 2-metric d represents the area of a triangle whose vertices are $x, y$ and $z$. Hsiao [2] have showed that contraction-like conditions on the operators in 2-metric spaces imply either the space is trivial or the operator is trivial.

In 1984, Dhage [3] introduced the concept of D-metric spaces. The situation for a D-metric space is quite different from 2-metric spaces. Geometrically, a D-metric $\mathrm{D}(\mathrm{x}, \mathrm{y}, \mathrm{z})$ represent the perimeter of the triangle with vertices $x, y$ and $z$ in $R^{2}$.

Recently, Mustafa and Sims [4] had shown that most of the results concerning Dhage's D-metric spaces are invalid and they introduced an improved version of the generalized metric space structure and called it as G-

${ }^{*}$ Corresponding author. metric spaces. For more details on G-metric spaces one can refer to the papers [4-7].

Now we give preliminaries and basic definitions which are used throughout the paper.

In 2006, Mustafa and Sims [5] introduced the concept of G-metric spaces as follows:

Definition 1.1. Let $X$ be a non-empty set and $G: X \times X \times X \rightarrow \mathbb{R}^{+}$be a function satisfying the following conditions :

(G1) $G(x, y, z)=0$ if $x=y=z$,

(G2) $0<G(x, x, y)$ for all $x, y \in X$ with $x \neq y$,

(G3) $G(x, x, y) \leq G(x, y, z)$ for all $x, y, z \in X$ with $z \neq y$,

(G4) $G(x, y, z)=G(x, z, y)=G(y, z, x)=$ ...(symmetry in all variables),

(G5) $G(x, y, z) \leq G(x, a, a)+G(a, y, z)$ for all $x, y, z, a \in X$ (rectangle inequality).

The function $\mathrm{G}$ is called a generalized metric or more specifically, a G-metric on $X$, and the pair $(X, G)$ is called a G-metric space.

Let $(X, G)$ be a G-metric space, $\left\{x_{n}\right\}$ a sequence of points in $X$. we say that $\left\{x_{n}\right\}$ is G-convergent to $x$ if $\lim _{m, n \rightarrow \infty} G\left(x, x_{n}, x_{m}\right)=0$, i.e., for each $\varepsilon>0$ there exists an $\mathrm{N}$ such that $G\left(x, x_{n}, x_{m}\right)<\varepsilon$ for all $m, n \geq N$. We call $x$ the limit of the sequence and write

$x_{n} \rightarrow x$ or $\lim _{n \rightarrow \infty} x_{n}=x$. 
Proposition 1.1. Let $(X, G)$ be a G-metric space. Then the following are equivalent:

1) $\left\{x_{n}\right\}$ is $G$ convergent to $x$,

2) $G\left(x_{n}, x_{n}, x\right) \rightarrow 0$ as $n \rightarrow \infty$,

3) $G\left(x_{n}, x, x\right) \rightarrow 0$ as $n \rightarrow \infty$,

4) $G\left(x_{m}, x_{n}, x\right) \rightarrow 0$ as $m, n \rightarrow \infty$.

Definition 1.2. Let $(X, G)$ be a G-metric space. A sequence $\left\{\mathrm{X}_{\mathrm{n}}\right\}$ is called G-Cauchy if, for each $\varepsilon>0$ there exist an $\mathrm{N} \in \mathbb{N}$ (set of positive integers) such that $G\left(x_{n}, x_{m}, x_{l}\right)<\varepsilon$ for all $n, m, l \geq N$.

Proposition 1.2. In a $\mathrm{G}$-metric space $(X, G)$ the following are equivalent:

1) The sequence $\left\{x_{n}\right\}$ is G-Cauchy,

2) for each $\varepsilon>0$ there exists an $N \in \mathbb{N}$ (set of positive integers) such that $G\left(x_{n}, x_{m}, x_{l}\right)<\varepsilon$ for all $n, m, l \geq N$.

Proposition 1.3. Let $(X, G)$ be a G-metric space. Then the function $G(x, y, z)$ is jointly continuous in all three of its variables.

Definition 1.3. A G-metric space $(X, G)$ is called a symmetric G-metric space if $G(x, y, y)=G(y, x, x)$ for all $x$, $y$ in $X$.

Proposition 1.4. Every G-metric space $(X, G)$ defines a metric space $\left(X, d_{G}\right)$ by

1) $d_{G}(x, y)=G(x, y, y)+G(y, x, x)$ for all $x, y \in X$.

If $(X, G)$ is a symmetric G-metric space, then

2) $d_{G}(x, y)=2 G(x, y, y)$ for all $x, y \in X$.

However, if $(X, G)$ is not symmetric, then it follows

from the $G$-metric properties that

3) $\frac{3}{2} G(x, y, y) \leq d_{G}(x, y) \leq 3 G(x, y, y)$

for all $x, y \in X$.

Proposition 1.5. A G-metric space $(X, G)$ is Gcomplete if and only if $\left(X, d_{G}\right)$ is a complete metric space.

Proposition 1.6. Let $(X, G)$ be a G-metric space. Then, for any $x, y, z, a$ in $X$ it follows that:

1) if $G(x, y, z)=0$, then $x=y=z$,

2) $G(x, y, z) \leq G(x, x, y)+G(x, x, z)$,

3) $G(x, y, y) \leq 2 G(y, x, x)$,

4) $G(x, y, z) \leq G(x, a, z)+G(a, y, z)$,

5) $G(x, y, z) \leq 2 / 3(G(x, a, a)+G(y, a, a)+G(z, a, a))$.

There has been a considerable interest to study common fixed point for a pair (or family) of mappings satisfying contractive conditions in metric spaces. Now, it is obvious that a fixed point of any map on metric spaces is a common fixed point of that map and identity map on the space. Several interesting and elegant results were obtained in this direction by various authors. It was the turning point in the "fixed point arena" when the notion of commutativity was used by Jungck [8] to obtain common fixed point theorems. While proving his result, Jungck [8] replaced identity map with a continuous map. Theorem 1.1 [8]. Let $T$ be a continuous mapping of a complete metric space $(X, d)$ into itself. Then $T$ has a fixed point in $X$ if there exist

$$
\begin{aligned}
& \alpha \in(0,1) \text { and a mapping } S: X \rightarrow X \text { which } \\
& \text { commutes with } T \text { and satisfies } S(X) \subset T(X) \\
& \text { and } d(S x, S y) \leq \alpha d(T x, T y) \text { for all } x, y \in X .
\end{aligned}
$$

From this result, it can be seen that a metrical common fixed point theorem generally involves conditions on commutativity, continuity, completeness and suitable containment of ranges of the involved maps besides an appropriate contraction condition and many authors in this domain are aimed at weakening one or more of these conditions.

In particular, now we look in the context of common fixed point theorems in G-metric spaces. Start with the following contraction conditions:

Let $T$ be a mapping from a complete metric space $(X, G)$ into itself and consider the following conditions:

$$
\begin{aligned}
& G(T x, T y, T z) \leq \alpha G(x, y, z) \\
& \text { for all } x, y, z \in X, \text { where } \alpha \in[0,1),
\end{aligned}
$$

It is clear that every self mapping $T$ of $X$ satisfying condition (1.1) is continuous. Now we focus to generalize the condition (1.1) for a pair of self maps $S$ and $T$ of $X$ in the following way:

$$
\begin{aligned}
& G(S x, S y, S z) \leq \alpha G(T x, T y, T z) \\
& \text { for all } x, y, z \in X, \text { where } \alpha \in[0,1),
\end{aligned}
$$

To prove the existence of common fixed points for (1.2), it is necessary to add additional assumptions such as construction of the sequence $\left\{x_{n}\right\}$ and some mechanism to obtain common fixed point. Most of the theorems in literature have followed a similar pattern of maps: 1) contraction; 2) continuity of functions (either one or both); and 3) commuting pair of mappings were given. In some cases condition 2) can be relaxed but condition 1) and 3) are unavoidable.

In 1986, Jungck [9] introduced the concept of compatibility in metric spaces as follows:

Two self-mappings $\mathrm{f}$ and $\mathrm{g}$ of a metric space $(X, d)$ are said to be compatible if

$\lim _{n \rightarrow \infty} d\left(f g x_{n}, g f x_{n}\right)=0$, whenever $\left\{x_{n}\right\}$ is a

sequence in $X$ such that $\lim _{n \rightarrow \infty} f x_{n}=\lim _{n \rightarrow \infty} g x_{n}=t$

for some $t \in X$. 


\section{Compatible Maps}

Recently, Choudhury et al. [10] introduced the notion of compatible maps in G-metric space as follows:

Let $f$ and $g$ be self maps of a G-metric space $(X, G)$. The maps $f$ and $g$ are said to be compatible map if there exists a sequence

$\left\{x_{n}\right\}$ such that $\lim _{n \rightarrow \infty} G\left(f g x_{n}, g f x_{n}, g f x_{n}\right)=0$

or $G\left(g f x_{n}, f g x_{n}, f g x_{n}\right)=0$, whenever $\left\{x_{n}\right\}$

is a sequence in $X$ such that

$$
\lim _{n \rightarrow \infty} f x_{n}=\lim _{n \rightarrow \infty} g x_{n}=t \text { for some } t \in X .
$$

\section{Example 2.1.}

Let $X=[-1,1]$ and let $G$ be the $G$-metric on $X$ defined as follows :

$G(x, y, z)=(|x-y|+|y-z|+|z-x|)$

for all $x, y, z \in X$. Then $(X, G)$ is a $G$-metric

space. Define $f(x)=x$ and $g x=x / 4$. Consider

the sequence $\left\{x_{n}\right\}=1 / n$. Clearly

$\lim _{n \rightarrow \infty} G\left(f g x_{n}, g f x_{n}, g f x_{n}\right)=0$ and $\lim _{n \rightarrow \infty} f x_{n}=$

$\lim _{n \rightarrow \infty} g x_{n}=0$ implies $f$ and $g$ are compatible maps.

Now we come to our main result for a pair of compatible maps.

Theorem 2.1. Let $(X, G)$ be a complete G-metric space. Let $f$ and $g$ be self mappings of $X$ satisfying the following conditions:

(2.1) $f(x) \subseteq g(x)$,

(2.2) $f$ or $g$ is continuous,

(2.3) $G(f x, f y, f z)$

$$
\leq k \max \left\{\begin{array}{l}
G(g x, g y, g z), G(g x, f x, f x), G(g x, f y, f y), \\
G(g x, f z, f z), G(g y, f y, f y), G(g y, f x, f x), \\
G(g y, f z, f z), G(g z, f z, f z), G(g z, f x, f x), \\
G(g z, f y, f y)
\end{array}\right\}
$$

for all $x, y, z \in X$, where $0 \leq k<1 / 4$.

Then $f$ and $g$ have a unique common fixed point in $X$ provided $f$ and $g$ are compatible maps.

Proof. Let $x_{0}$ be an arbitrary point in $X$. By (2.1), one can choose a point

$x_{1}$ in $X$ such that $f x_{0}=g x_{1}$. In general

one can choose $x_{n+1}$ such that $y_{n}=f x_{n}=g x_{n+1}$,

$n=0,1,2, \cdots$
From (2.3), we have

$$
\text { (2.4) } G\left(f x_{n}, f x_{n+1}, f x_{n+1}\right)
$$

$$
\leq k \max \left\{\begin{array}{l}
G\left(g x_{n}, g x_{n+1}, g x_{n+1}\right), G\left(g x_{n}, f x_{n}, f x_{n}\right), \\
G\left(g x_{n}, f x_{n+1}, f x_{n+1}\right), G\left(g x_{n}, f x_{n+1}, f x_{n+1}\right), \\
G\left(g x_{n+1}, f x_{n+1}, f x_{n+1}\right), G\left(g x_{n+1}, f x_{n}, f x_{n}\right), \\
G\left(g x_{n+1}, f x_{n+1}, f x_{n+1}\right), G\left(g x_{n+1}, f x_{n+1}, f x_{n+1}\right), \\
G\left(g x_{n+1}, f x_{n}, f x_{n}\right), G\left(g x_{n+1}, f x_{n+1}, f x_{n+1}\right)
\end{array}\right\}
$$

or

$$
\begin{aligned}
& G\left(y_{n}, y_{n+1}, y_{n+1}\right) \leq k \max \left\{G\left(y_{n-1}, y_{n}, y_{n}\right),\right. \\
& \left.G\left(y_{n-1}, y_{n+1}, y_{n+1}\right), G\left(y_{n}, y_{n+1}, y_{n+1}\right)\right\}
\end{aligned}
$$

\section{Case 1.}

$$
\text { If } \begin{aligned}
\max & \left\{G\left(y_{n-1}, y_{n}, y_{n}\right), G\left(y_{n-1}, y_{n+1}, y_{n+1}\right),\right. \\
& \left.G\left(y_{n}, y_{n+1}, y_{n+1}\right)\right\}=G\left(y_{n-1}, y_{n}, y_{n}\right)
\end{aligned}
$$

then, using (2.3), we get

$G\left(y_{n}, y_{n+1}, y_{n+1}\right) \leq k G\left(y_{n-1}, y_{n}, y_{n}\right)$, Continuing in the same way, we have

$$
G\left(y_{n}, y_{n+1}, y_{n+1}\right) \leq k^{n} G\left(y_{0}, y_{1}, y_{1}\right) \text {. Therefore, }
$$

for all $n, m \in N, n<m$ and by(G5), we have

$$
\begin{aligned}
& G\left(y_{n}, y_{m}, y_{m}\right) \leq G\left(y_{n}, y_{n+1}, y_{n+1}\right) \\
& +G\left(y_{n+1}, y_{n+2}, y_{n+2}\right)+\cdots+G\left(y_{m-1}, y_{m}, y_{m}\right) \\
& \leq\left(k^{n}+k^{n+1}+\ldots+k^{m-1}\right) G\left(y_{0}, y_{1}, y_{1}\right) \\
& \leq \frac{k^{n}}{1-k} G\left(y_{0}, y_{1}, y_{1}\right) .
\end{aligned}
$$

Letting as $n, m \rightarrow \infty$, we have

$$
\lim _{n \rightarrow \infty} G\left(y_{n}, y_{m}, y_{m}\right)=0 \text {. Thus }\left\{y_{n}\right\} \text { is a } G-
$$

Cauchy sequence in $X$.

\section{Case 2.}

$$
\text { If } \begin{aligned}
\max & \left\{G\left(y_{n-1}, y_{n}, y_{n}\right), G\left(y_{n-1}, y_{n+1}, y_{n+1}\right),\right. \\
& \left.G\left(y_{n}, y_{n+1}, y_{n+1}\right)\right\}=G\left(y_{n-1}, y_{n+1}, y_{n+1}\right)
\end{aligned} .
$$

From (2.3) and using (G5), one obtains

$$
\begin{aligned}
& G\left(y_{n}, y_{n+1}, y_{n+1}\right) \leq k G\left(y_{n-1}, y_{n+1}, y_{n+1}\right) \\
& \leq k\left(G\left(y_{n-1}, y_{n}, y_{n}\right)+G\left(y_{n}, y_{n+1}, y_{n+1}\right)\right)
\end{aligned}
$$

implies

$$
G\left(y_{n}, y_{n+1}, y_{n+1}\right) \leq \frac{k}{1-k} G\left(y_{n-1}, y_{n}, y_{n}\right),
$$

i.e., $G\left(y_{n}, y_{n+1}, y_{n+1}\right) \leq q G\left(y_{n-1}, y_{n}, y_{n}\right)$, where

$q=\frac{k}{1-k}$ and $q<1$ as $0 \leq k<1 / 4$ 
In view of Case 1 , we have $\left\{y_{n}\right\}$ is G-Cauchy sequence in $X$.

Case 3.

$$
\begin{aligned}
& \text { If } \max \left\{G\left(y_{n-1}, y_{n}, y_{n}\right), G\left(y_{n-1}, y_{n+1}, y_{n+1}\right),\right. \\
& \left.G\left(y_{n}, y_{n+1}, y_{n+1}\right)\right\}=G\left(y_{n}, y_{n+1}, y_{n+1}\right)
\end{aligned}
$$

then, from (2.3), one obtains

$$
\begin{aligned}
& G\left(y_{n}, y_{n+1}, y_{n+1}\right) \leq k G\left(y_{n}, y_{n+1}, y_{n+1}\right) \\
& \text { a contradiction as } k<1 / 4 .
\end{aligned}
$$

Hence in all cases the sequence $\left\{y_{n}\right\}$ is a G-Cauchy sequence in $X$.

Since $(X, G)$ is complete G-metric space, therefore, there exists a point $u \in X$ such that $\lim _{n \rightarrow \infty} y_{n}=u$, we have $\lim _{n \rightarrow \infty} y_{n}=\lim _{n \rightarrow \infty} f x_{n}=\lim _{n \rightarrow \infty} g x_{n+1}=u$. Since one of the maps $f$ or $g$ is continuous, for definiteness one can assume that $g$ is continuous, therefore

$\lim _{n \rightarrow \infty} g f x_{n}=\lim _{n \rightarrow \infty} g g x_{n}=g u$. Further, $f$ and $g$ are compatible, therefore, $\lim _{n \rightarrow \infty} G\left(g f x_{n}, f g x_{n}, f g x_{n}\right)=0$ implies

$\lim _{n \rightarrow \infty} f g x_{n}=g u$. Now we claim that $g u=u$, from (2.3), we have

$$
\begin{aligned}
& G\left(f g x_{n}, f x_{n}, f x_{n}\right) \leq k \max \\
& \left\{\begin{array}{l}
G\left(g g x_{n}, g x_{n}, g x_{n}\right), G\left(g g x_{n}, f g x_{n}, f g x_{n}\right), \\
G\left(g g x_{n}, f x_{n}, f x_{n}\right), G\left(g g x_{n}, f x_{n}, f x_{n}\right), \\
G\left(g x_{n}, f x_{n}, f x_{n}\right), G\left(g x_{n}, f g x_{n}, f g x_{n}\right), \\
G\left(g x_{n}, f x_{n}, f x_{n}\right), G\left(g x_{n}, f x_{n}, f x_{n}\right), \\
G\left(g x_{n}, f g x_{n}, f g x_{n}\right), G\left(g x_{n}, f x_{n}, f x_{n}\right)
\end{array}\right\} .
\end{aligned}
$$

Proceeding limit as $n \rightarrow \infty$, and using Proposition 1.6, we have

$$
\begin{aligned}
& G(g u, u, u) \leq k \max \{G(g u, u, u), G(u, g u, g u)\} \\
& \leq k \max \{G(g u, u, u), 2 G(g u, u, u)\} \\
& =2 k G(g u, u, u), \text { a contradiction as } k<1 / 4
\end{aligned}
$$

Hence $g u=u$, next we will show that $g u=f u=u$, for this purpose we put $x=x_{n}, y=z=u$ in (2.3), we have

$$
\begin{aligned}
& G\left(f x_{n}, f u, f u\right) \leq k \max \\
& \left\{\begin{array}{l}
G\left(g x_{n}, g u, g u\right), G\left(g x_{n}, f x_{n}, f x_{n}\right), G\left(g x_{n}, f u, f u\right) \\
G\left(g x_{n}, f u, f u\right), G(g u, f u, f u), G\left(g u, f x_{n}, f x_{n}\right) \\
G(g u, f u, f u), G(g u, f u, f u), G\left(g u, f x_{n}, f x_{n}\right) \\
G(g u, f u, f u)
\end{array}\right\}
\end{aligned}
$$

Proceeding limit as $n \rightarrow \infty$, we have $G(u, f u, f u) \leq k G(u, f u, f u)$, a contradiction as $k<1 / 4$ Hence $f u=g u=u$. Thus $u$ is a common fixed point of f and $g$.

Uniqueness. We assume that $u_{1}(\neq u)$ be another common fixed point of $f$ and $g$. Then

$G\left(u_{1}, u, u\right)>0$ and

$$
G\left(u_{1}, u, u\right)=G\left(f u_{1}, f u, f u\right) \leq k \max
$$$$
\left\{\begin{array}{l}
G\left(g u_{1}, g u, g u\right), G\left(g u_{1}, f u_{1}, f u_{1}\right), G\left(g u_{1}, f u, f u\right) \\
G\left(g u_{1}, f u, f u\right), G(g u, f u, f u), G\left(g u, f u_{1}, f u_{1}\right) \\
G(g u, f u, f u), G(g u, f u, f u), G\left(g u, f u_{1}, f u_{1}\right) \\
G(g u, f u, f u)
\end{array}\right\}
$$

By using Proposition 1.6, we have

$$
\begin{aligned}
& G\left(u_{1}, u, u\right) \leq k \max \left\{G\left(u_{1}, u, u\right), G\left(u, u_{1}, u_{1}\right)\right\} \\
& \leq k \max \left\{G\left(u_{1}, u, u\right), 2 G\left(u_{1}, u, u\right)\right\} \\
& =2 k G\left(u_{1}, u, u\right), \text { a contradiction as } k<1 / 4 .
\end{aligned}
$$

This demands that $u_{1}=u$. Hence uniqueness follows.

Example 2.2. Let $X=[-1,1]$ and let $G$ be the G-metric on $X$ defined as follows:

$$
G(x, y, z)=(|x-y|+|y-z|+|z-x|)
$$

for all $x, y, z \in X$.

Then $(X, G)$ is a $G$-metric space. Define $f(x)=$ $x / 9$ and $g x=x$. Then $f(X) \subseteq g(X)$

Also, inequality (2.3) holds for all $x, y, z$ in $X$ and 0 is the unique common fixed point of $f$ and $g$.

Corollary 2.1. Let $(X, G)$ be a complete G-metric space and let $f$ and $g$ be compatible mapping of $X$ satisfying (2.1), (2.2) and the following condition:

$$
G(f x, f y, f z) \leq q G(g x, g y, g z)
$$

for every $x, y, z$ in $X$ and $0<q<1$.

Then $f$ and $g$ have a unique common fixed point in $X$.

Proof: Proof follows easily from Theorem 2.1.

\section{Weakly Compatible Maps}

In 1996, Jungck [11] introduce the notion of weakly compatible mappings as follows:

Definition 3.1. Two maps $f$ and $g$ are said to be weakly compatible if they commute at coincidence points.

Example 3.1. Let $X=[0,3]$.

Define $f, g:[0,3] \rightarrow[0,3]$ by

$$
f(x)=\left\{\begin{array}{l}
x \text { if } x \in[0,1) \\
3 \text { if } x \in[1,3]
\end{array} ; g(x)= \begin{cases}3-x & \text { if } x \in[0,1) \\
3 & \text { if } x \in[1,3]\end{cases}\right.
$$

Then for any $x \in[1,3], x$ is a coincidence point and $f g x$ $=g f x$, showing that $f, g$ are weakly compatible maps on $[0$, 3].

Theorem 3.1. Let $f$ and $g$ be weakly compatible self 
maps of a G-metric space $(X, G)$ satisfying (2.1) and (2.3) and the following condition:

(3.1) any one of the subspace $f(X)$ or $g(X)$ is complete; Then $f$ and $g$ have a unique common fixed point in $X$.

Proof. From Theorem 2.1, we conclude that $\left\{y_{n}\right\}$ is a G-Cauchy sequence in $X$. Since either $f(X)$ or $g(X)$ is complete, for definiteness assume that $g(X)$ is complete subspace of $X$ then the subsequence of $\left\{y_{n}\right\}$ must get a limit in $g(X)$. Call it be $t$. Let $u \in g^{-1} t$. Then $g u=t$ as $\left\{y_{n}\right\}$ is a G-Cauchy sequence containing a convergent subsequence, therefore the sequence $\left\{y_{n}\right\}$ also convergent implying thereby the convergence of subsequence of the convergent sequence. Now we show that $f u=t$.

On setting $x=x_{n}, y=u$ and $z=u$, in (2.3), we have

$$
\begin{aligned}
& G\left(f x_{n}, f u, f u\right) \leq k \max \\
& \left\{\begin{array}{l}
G\left(g x_{n}, g u, g u\right), G\left(g x_{n}, f x_{n}, f x_{n}\right), G\left(g x_{n}, f u, f u\right), \\
G\left(g x_{n}, f u, f u\right), G(g u, f u, f u), G\left(g u, f x_{n}, f x_{n}\right), \\
G(g u, f u, f u), G(g u, f u, f u), G\left(g u, f x_{n}, f x_{n}\right), \\
G(g u, f u, f u)
\end{array}\right\}
\end{aligned}
$$

Proceeding $\lim i t$ as $n \rightarrow \infty$, we have

$G(t, f u, f u) \leq k G(t, f u, f u)$, a contradiction as $k<1 / 4$.

Hence $f u=g u=t$. Thus $u$ is a coincident point of $f$ and $g$. Since $f$ and $g$ are weakly compatible, it follows that $f g u=$ gfu, i.e., $f t=g t$.

We now show that $f t=t$. Suppose that $f t \neq t$, therefore $G(f t, t, t)>0$. Now put on setting $x=t, y=u, z=u$, in (2.3), we have

$$
\begin{aligned}
& G(f t, f u, f u) \\
& \leq k \max \left\{\begin{array}{l}
G(g t, g u, g u), G(g t, f t, f t), G(g t, f u, f u), \\
G(g t, f u, f u), G(g u, f u, f u), G(g u, f t, f t), \\
G(g u, f u, f u), G(g u, f u, f u), G(g u, f t, f t), \\
G(g u, f u, f u)
\end{array}\right\}
\end{aligned}
$$

By using Proposition 1.6, we have

$$
\begin{aligned}
& G(f t, t, t) \leq k \max \{G(f t, t, t), G(t, f t, f t)\} \\
& \leq k \max \{G(f t, t, t), 2 G(f t, t, t)\} \\
& =2 k G(f t, t, t), \text { a contradiction as } k<1 / 4
\end{aligned}
$$

This demands that $f t=t=g t$, i.e., $t$ is common fixed point of $f$ and $g$. Uniqueness follows easily.

\section{Property (E.A.) in G-Metric Spaces}

Recently, Amari and Moutawakil [12] introduced a generalization of non compatible maps as property (E.A.) in metric spaces as follows:

Definition 4.1. Let $A$ and $S$ be two self-maps of a metric space $(X, d)$. The pair $(A, S)$ is said to satisfy property
(E.A.), if there exists a sequence $\left\{X_{n}\right\}$ in $X$ such that $\lim _{n \rightarrow \infty} A x_{n}=\lim _{n \rightarrow \infty} S x_{n}=t$ for some $t \in X$.

In similar mode, we use property (E.A.) in G-metric spaces.

Example 4.1 [12]. Let

$$
\begin{aligned}
& X=[0, \infty) . \text { Define } S, T: X \rightarrow X \text { by } T x=\frac{X}{4} \text { and } \\
& S x=\frac{3 X}{4} \text { for all } x \in X . \text { Consider } x_{n}=\frac{1}{n} . \text { Clearly } \\
& \lim _{n \rightarrow \infty} S x_{n}=\lim _{n \rightarrow \infty} T x_{n}=0 .
\end{aligned}
$$

Then $S$ and $T$ satisfy property (E.A.).

\section{Example 4.2 [12]. Let}

$$
\begin{aligned}
& X=[2, \infty) . \text { Define } S, T: X \rightarrow X \text { by } \\
& T x=x+1 \text { and } S x=2 x+1 \text { for all } x \in X .
\end{aligned}
$$

Suppose that the property (E.A.) holds. Then, there exists in $X$ a sequence $\left\{x_{n}\right\}$ satisfying $\lim _{n \rightarrow \infty} S x_{n}=\lim _{n \rightarrow \infty} T x_{n}=z$ for some $z \in X$. Therefore, $\lim _{n \rightarrow \infty} S x_{n}=\lim _{n \rightarrow \infty} T x_{n}=z$ for some $z \in X$.

$\lim _{n \rightarrow \infty} x_{n}=z-1$ and $\lim _{n \rightarrow \infty} x_{n}=\frac{z-1}{2}$. Thus, $z=1$, a contradiction, since 1 is not contained in $X$. Hence $S$ and $T$ do not satisfy property (E.A.).

Notice that weakly compatible and property (E.A.) are independent to each other (for detail see, [13]).

Example 4.3. Let $X=\mathbb{R}^{+}$and $(X, d)$ be the usual metric space. Define $f, g: X \rightarrow X$ by

$f x=\left\{\begin{array}{l}0, \text { if } x \in(0,1], \\ 1, \text { if } x=0 \text { and } x>1\end{array} ; g x=[x]\right.$, the greatest integer that is less than or equal to $x$, for all $x \in X$. Consider a sequence $\left\{x_{n}\right\}=\{1+1 / n\}, n \geq 2$ in $(1,2)$, then we have $\lim _{n \rightarrow \infty} f x_{n}=1=\lim _{n \rightarrow \infty} g x_{n}$. Similarly for the sequence $\left\{y_{n}\right\}=\{1-1 / n\}, n \geq 2$ in $(0,1)$, then we have $\lim _{n \rightarrow \infty} f y_{n}=0=\lim _{n \rightarrow \infty} g y_{n}$.

Thus the pair $(f, g)$ satisfies property (E.A.). However, $f$ and $g$ are not weakly compatible as each $u_{1} \in(0,1)$ and $u_{2} \in(1,2)$ are coincidence points of $f$ and $g$, where they do not commute. Moreover, they commute at $x=0,1,2, \cdots$ but none of these points are coincidence points of $f$ and $g$. Thus we can conclude that, property (E.A.) does not imply weak compatibility.

Now we prove a common fixed point theorem for a pair of weakly compatible maps along with property (E.A.).

Theorem 4.1. Let $f$ and $g$ be self maps of a G-metric space $(X, G)$ satisfying $(2.3)$ and the following con- 
ditions:

(4.1) $f$ and $g$ satisfy property (E.A.),

(4.2) $g(X)$ is a closed subspace of $X$.

Then $f$ and $g$ have a unique common fixed point in $X$ provided $f$ and $g$ are weakly compatible self maps.

Proof. Since $f$ and g satisfy property (E.A.), therefore, there exists a sequence $\left\{x_{n}\right\}$ in $X$ such that

$\lim _{n \rightarrow \infty} f x_{n}=\lim _{n \rightarrow \infty} g x_{n}=u$ for some $u \in X$. Since $g(X)$

is a closed subspace of $X$, therefore, $\lim _{n \rightarrow \infty} f x_{n}=u=$

$g a=\lim _{n \rightarrow \infty} g x_{n}$ for some $a \in X \Rightarrow u=g a \in X$.

We claim that $f a=g a=u$. From(2.3), we have

$$
\begin{aligned}
& G\left(f x_{n}, f a, f a\right) \leq k \max \\
& \left\{\begin{array}{l}
G\left(g x_{n}, g a, g a\right), G\left(g x_{n}, f x_{n}, f x_{n}\right), G\left(g x_{n}, f a, f a\right) \\
G\left(g x_{n}, f a, f a\right), G(g a, f a, f a), G\left(g a, f x_{n}, f x_{n}\right) \\
G(g a, f a, f a), G(g a, f a, f a), G\left(g a, f x_{n}, f x_{n}\right) \\
G(g a, f a, f a)
\end{array}\right\}
\end{aligned}
$$

Proceeding limit as $n \rightarrow \infty$, we have

$G(u, f a, f a) \leq k G(u, f a, f a)$, a contradiction as $k<1 / 4$.

Hence $f a=g a=u$. Thus $u$ is a coincident point of $f$ and $g$.

Since $f$ and $g$ are weakly compatible maps.

Rest of the proof follows easily from Theorem 3.1.

Corollary 4.1. Let $f$ and $g$ be self maps of a G-metric space $(X, G)$ satisfying (4.1) and (4.2) and the following condition:

$$
\begin{aligned}
& G(f x, f y, f z) \leq q G(g x, g y, g z) \text { for all } x, y, z \in X \\
& \text { and } 0 \leq q<1 .
\end{aligned}
$$

Then $f$ and $g$ have a unique common fixed point in $X$ provided $f$ and $g$ be weakly compatible self maps.

Proof. Proof follows easily from Theorem 4.1.

\section{Example4.1.}

Let $X=[0,2]$ and let $G$ be the $G$-metric on $X$ defined as follows :

Define $G(x, y, z)=(|x-y|+|y-z|+|z-x|)$

and $f, g: X \rightarrow X$ as follows:

$$
f(x)=\left\{\begin{array}{l}
0, \text { if } x \in[0,1), \\
1, \text { if } x \in[1,2] .
\end{array} ; g(x)=\left\{\begin{array}{l}
0, \text { if } x \in[0,1), \\
2, \text { if } x \in[1,2] .
\end{array}\right.\right.
$$

Consider $x_{n}=1 / n$. Clearly $\lim _{n \rightarrow \infty} f x_{n}=\lim _{n \rightarrow \infty} g x_{n}=0$.

Then $f$ and $g$ satisfy property (E.A.). Also $f(X)=\{0,1\}$ and $g(X)=\{0,2\}$.

Here we note that neither $f(X)$ is contained in $g(X)$ nor $g(X)$ is contained in $f(X)$ and Corollary 4.1 holds for $1 / 2 \leq \mathrm{q}<1$.

Remark 4.1. It can be observed that a pair of maps enjoying property (E.A.) relaxes the required containment of range of one mapping into the range of other which is utilized to construct the sequence of joint iterates. Moreover, it buys containment of ranges without any continuity requirements besides minimizes the commutativity conditions of the maps to the commutativity at their points of coincidence and it also allows replacing the completeness requirement of the space with a more natural condition of closeness of the range.

\section{5. (CLRg) Property in G-Metric Spaces}

Recently, Sintunavarat and Kumam [14] introduced a new property which is so called "Common Limit in the Range of g property" (i.e., (CLRg ) property) and defined as follow:

Definition 5.1 [14]. Suppose that $(X, d)$ is a metric space and $f, g: X \rightarrow X$. Two mappings $f$ and $g$ are said to satisfy the common limit in the range of $g$ property if there exists a sequence $\left\{x_{n}\right\}$ in $X$ such that

$$
\lim _{n \rightarrow \infty} f x_{n}=\lim _{n \rightarrow \infty} g x_{n}=g x \text { for some } x \in X .
$$

The common limit in the range of $g$ property will be denoted by the (CLRg) property.

\section{Example 5.1 [14].}

Let $X=[0, \infty)$ be the usual mrtric space. Define $f, g: X \rightarrow X$ by $f x=x+1$ and $g x=2 x$ for all $x \in X$. Consider the sequence $\left\{x_{n}\right\}=\left\{1+\frac{1}{n}\right\}$. Since $\lim _{n \rightarrow \infty} f x_{n}=\lim _{n \rightarrow \infty} g x_{n}=g 1=2$, therefore, $f \& g$ satisfy (CLRg) property.

\section{Example 5.2 [14].}

Let $X=[0, \infty)$ be the usual mrtric space. Define

$$
f, g: X \rightarrow X \text { by } f x=\frac{x}{4} \text { and } g x=\frac{3 x}{4} \text { for all }
$$

$x \in X$. Consider the sequence $\left\{x_{n}\right\}=\left\{\frac{1}{n}\right\}$.

Since $\lim _{n \rightarrow \infty} f x_{n}=\lim _{n \rightarrow \infty} g x_{n}=g 0=0$,

therefore, $f \& g$ satisfy(CLRg) property.

In similar mode, we use (CLRg) property in G-metric spaces.

Theorem 5.1. Let $f$ and $g$ be self maps of a G-metric space $(X, G)$ satisfying $(2.3)$ and the following condition: (5.1) $f$ and $g$ satisfy (CLRg) property,

Then $f$ and $g$ have a unique common fixed point in $X$ provided $f$ and $g$ are weakly compatible maps.

Proof. Since $f$ and $g$ satisfy (CLRg) property, therefore, there exists a sequence $\left\{x_{n}\right\}$ in $X$ such that 


$$
\lim _{n \rightarrow \infty} f x_{n}=\lim _{n \rightarrow \infty} g x_{n}=g a=u \text { for some } a, u \in X .
$$

We claim that $f a=g a=u$. From (2.3), we have

$$
\begin{aligned}
& G\left(f x_{n}, f a, f a\right) \leq k \max \\
& \left\{\begin{array}{l}
G\left(g x_{n}, g a, g a\right), G\left(g x_{n}, f x_{n}, f x_{n}\right), G\left(g x_{n}, f a, f a\right), \\
G\left(g x_{n}, f a, f a\right), G(g a, f a, f a), G\left(g a, f x_{n}, f x_{n}\right), \\
G(g a, f a, f a), G(g a, f a, f a), G\left(g a, f x_{n}, f x_{n}\right), \\
G(g a, f a, f a)
\end{array}\right\}
\end{aligned}
$$

Proceeding limit as $n \rightarrow \infty$, we have $G(u, f a, f a) \leq k G(u, f a, f a)$, a contradiction as $k<1 / 4$ Hence $f a=g a=u$. Thus $u$ is a coincident point of $f$ and $g$. Since $f$ and $g$ are weakly compatible maps.

Rest of the Proof follows easily from Theorem 3.1.

Remark 5.1. We note that (CLRg) property relaxes the condition of closeness of range.

\section{Acknowledgements}

Authors (Sanjay Kumar, Naresh Kumar and S. K. Garg) would like to acknowledge University Grants Commission, Delhi, India for providing financial grant for the Major Research Project under Ref. No. 39-41/2010(SR).

\section{REFERENCES}

[1] S. Gahler, "2-Metrices Raume und Ihre Topologische Struktur," Mathematische Nachrichten, Vol. 26, 1963, pp. 115-148. doi:10.1002/mana.19630260109

[2] C. R. Hsiao, "A Property of Contraction Type Mappings in 2-Metric Spaces," Janabha, Vol. 16, 1986, pp. 223229.

[3] B. C. Dhage, "Generalized Metric Spaces and Mappings with Fixed Point," Bulletin of Calcutta Mathematical Society, Vol. 84, 1992, pp. 329-336.

[4] Z. Mustafa and B. Sims, "Some Remarks Concerning D-Metric Spaces," Proceedings of International Confer- ence on Fixed Point Theory and Applications, Yokohama Publishers, Valencia, Vol. 13, No. 19, 2004, pp. 189-198.

[5] Z. Mustafa and B. Sims, "A New Approach to a Generalized Metric Spaces," Journal of Nonlinear and Convex Analysis, Vol. 7, No. 2, 2006, pp. 289-297.

[6] Z. Mustafa, H. Obiedat and F. Awawdeh, "Some Fixed Point Theorems for Mappings on Complete G-Metric Spaces," Fixed Point Theory and Applications, 2008, Article ID: 18970, 12 p. doi:10.1155/2008/189870

[7] Z. Mustafa, W. Shatanawi and M. Bataineh, "Existence of Fixed Points Results in G-Metric Spaces," International Journal of Mathematics and Mathematical Sciences, 2009, Article ID: 283028, 10 p. doi:10.1155/2009/283028

[8] G., Jungck, "Commuting Mappings and Fixed Point," American Mathematical Monthly, Vol. 83, 1976, pp. 261263. doi: $10.2307 / 2318216$

[9] G. Jungck, "Compatible Mappings and Common Fixed Points," International Journal of Mathematics and Mathematical Sciences, Vol. 9, No. 4, 1986, pp. 771-779. doi:10.1155/S0161171286000935

[10] B. S. Choudhury, S. Kumar, Asha and K. Das, "Some Fixed Point Theorems in G-Metric Spaces," Nonlinear Science Letters, Vol. 1, No. 1, 2012, pp. 25-31.

[11] G., Jungck, "Common fixed Points for Non-Continuous Non-Self Mappings on Non-Metric Spaces," Far East Journal of Mathematical Sciences, Vol. 4, No. 2, 1996, pp. 199-215.

[12] M. Aamri and D. E. I. Moutawakil, "Some New Common Fixed Point Theorems under Strict Contractive Conditions," Journal of Mathematical Analysis and Applications, Vol. 270, 2002, pp. 181-188. doi:10.1016/S0022-247X(02)00059-8

[13] H. K. Pathak, R. Rodriguez-Lopez and R. K. Verma, “A Common Fixed Point Theorem Using Implicit Relation and E.A. Property in Metric Spaces," Filomat, Vol. 21, No. 2, 2007, pp. 211-234. doi:10.2298/FIL0702211P

[14] W. Sintunavarat and P. Kumam, "Common Fixed Point Theorems for a Pair of Weakly Compatible Mappings in Fuzzy Metric Spaces," Journal of Applied Mathematics, 2011, Article ID: 637958, 14 p. doi:10.1155/2011/637958 